\title{
Prognostic role of diffusion weighted and dynamic contrast-enhanced MRI in loco- regionally advanced head and neck cancer treated with concomitant chemoradiotherapy
}

\author{
Manca Garbajs ${ }^{1}$, Primoz Strojan², Katarina Surlan-Popovic ${ }^{1}$ \\ ${ }^{1}$ Institute of Clinical Radiology, University Medical Centre Ljubljana, Slovenia \\ 2 Division of Radiation Oncology, Institute of Oncology, Ljubljana, Slovenia
}

Radiol Oncol 2019; 53(1): 39-48.

Received 23 January 2019

Accepted 04 February 2019

Correspondence to: Manca Garbajs, M.D., Institute of Clinical Radiology, University Medical Centre, Zaloška c. 7, SI-1000 Ljubljana, Slovenia. Phone: + 38640212 226; E-mail: manca.garbajs@kclj.si

Disclosure: No potential conflicts of interest were disclosed.

Background. In the study, the value of pre-treatment dynamic contrast-enhanced (DCE) and diffusion weighted (DW) MRI-derived parameters as well as their changes early during treatment was evaluated for predicting diseasefree survival (DFS) and overall survival (OS) in patients with locoregionally advanced head and neck squamous carcinoma (HNSCC) treated with concomitant chemoradiotherapy (CCRT) with cisplatin.

Patients and methods. MRI scans were performed in 20 patients with locoregionally advanced HNSCC at baseline and after 10 Grays (Gy) of CCRT. Tumour apparent diffusion coefficient (ADC) and DCE parameters (volume transfer constant [ $\left.\mathrm{K}_{\text {trans }}\right]$, extracellular extravascular volume fraction $\left[\mathrm{V}_{\mathrm{e}}\right]$, and plasma volume fraction $\left[\mathrm{V}_{\mathrm{p}}\right]$ ) were measured. Relative changes in parameters from baseline to $10 \mathrm{~Gy}$ were calculated. Univariate and multivariate Cox regression analysis were conducted. Receiver operating characteristic (ROC) curve analysis was employed to identify parameters with the best diagnostic performance.

Results. None of the parameters was identified to predict for DFS. On univariate analysis of OS, lower pre-treatment $\operatorname{ADC}(p=0.012)$, higher pre-treatment $K_{\text {trans }}(p=0.026)$, and higher reduction in $K_{\text {trans }}(p=0.014)$ from baseline to $10 \mathrm{~Gy}$ were identified as significant predictors. Multivariate analysis identified only higher pre-treatment $K_{\text {trans }}(p=0.026 ; 95 \%$ Cl: $0.000-0.132)$ as an independent predictor of OS. At ROC curve analysis, pre-treatment $\mathrm{K}_{\text {trans }}$ yielded an excellent diagnostic accuracy (area under curve [AUC] $=0.95$, sensitivity 93.3\%; specificity $80 \%$ ).

Conclusions. In our group of HNSCC patients treated with cisplatin-based CCRT, pre-treatment $\mathrm{K}_{\text {trans }}$ was found to be a good predictor of OS.

Key words: dynamic contrast-enhanced MRl; diffusion-weighted imaging; overall survival; squamous cell head and neck cancer; concomitant chemoradiotherapy

\section{Introduction}

Head and neck squamous carcinoma (HNSCC) is the seventh most commonly diagnosed cancer and the sixth most common cause of cancer death worldwide. ${ }^{1}$ In a significant portion of patients with loco-regionally advanced disease, platinbased concomitant chemo-radiotherapy (cCRT) is the mainstay of treatment. ${ }^{2,3}$ However, current treatment options remain suboptimal, since around $50 \%$ of patient experience tumour recurrence ${ }^{4,5}$ with the pathohistological evidence of residual regional lymph node metastases in up to $26 \%$ of patients with clinically determined complete response after cCRT. ${ }^{6}$ In addition, the reported 5-year overall survival (OS) rates are still well below $50 \% .^{7}$ In this scenario, improving prognostic stratification either before or early during cCRT would allow for pos- 
sible modification of treatment regime in order to improve clinical outcomes and survival rates.

Multi-parametric imaging with functional imaging techniques has recently been used to evaluate biological properties of malignant tumours as changes at a cellular level typically occur prior to morphological changes. CT perfusion (CTP) has already been reported to provide a valuable information on tumour vascularity. ${ }^{8-10}$ In recent years, diffusion-weighted (DW) MR imaging (MRI) and dynamic contrast-enhanced MRI (DCE) have been used for assessing tumour biology and to predict tumour response and survival rates in patients with HNSCC. DWI is a widely used technique that allows for non-invasive measurement of the Brownian motion of water molecules by calculating the apparent diffusion coefficient (ADC) which reflects tumour cellularity and tissue microstructure. ${ }^{11}$

Dynamic contrast-enhanced MRI (DCE-MRI) can assess the flow of blood through vessels in scanned tissues and therefore enables non-invasive characterization of tumour vascularity and perfusion. ${ }^{12}$ Due to lack of standardization of data acquisition and analysis ${ }^{12}$, the results of studies assessing the value of DCE-MRI derived parameters are still scarce and contradictory. ${ }^{13-15}$ In addition, studies addressing prognostic values of DCE-MRI parameters acquired early during treatment are lacking.

To the best of our knowledge, there is no data in the literature that would adress the utility of combined DWI and DCE-MRI derived parameters before and early during treatment as prognostic factors for survival in HNSCC. Therefore, the objective of current study was to evaluate the prognostic value of imaging parameters from DWI and DCE MRI at baseline and early during treatment in loco-regionally advanced HNSCC patients treated with cisplatin-based cCRT.

\section{Patients and methods}

\section{Patients and treatment}

The study was approved by the National Medical Ethics Committee of the Republic of Slovenia (No. $22 \mathrm{k} / 03 / 13$ ) and written informed consent was obtained from all patients. Twenty patients with locally and/or regionally advanced (stages III-IVB) and histologically proven p16/HPV-negative SCC of the oro- or hypopharynx were enrolled in the study. p16/HPV status was assessed as described elsewhere. ${ }^{16}$ All patients were treated with cisplatin-based cCRT as recommended by the institutional multidisciplinary tumour board.
Patients were irradiated with a 6-MV linear accelerator photon beam using a concomitant boost intensity-modulated radiation technique. The dose to the primary tumour and enlarged lymph nodes was $70 \mathrm{~Gy}$ (gross tumour volume with a margin to compensate microscopic tumour extensions), whereas elective doses of $63 \mathrm{~Gy}$ (intermediate-risk volume - around larger nodes and non-palpable but radiologically suspicious nodes) and 56 Gy (low-risk volume) were applied for neck regions of probable microscopic disease in daily fractions of 2.0, 1.8 and 1.6 Gy, respectively, over 7 weeks. During radiotherapy, cisplatin in a dose of $40 \mathrm{mg} /$ $\mathrm{m}^{2} /$ week was concurrently administered. No antiangiogenic therapy was added to the protocol.

During therapy, toxicity was monitored on a weekly basis. After treatment completion, patients were examined for toxicity and tumour response every 2 to 3 months in the first and second year and every six months thereafter.

\section{MR imaging protocol}

All patients underwent three MR examinations with DWI and DCE-MRI: (1) 0-7 days before the treatment; (2) after 1 week (i.e., after the fifth fraction of radiotherapy, i.e. $10 \mathrm{~Gy}$ ) and (3) 2.5-3 months after the completion of cCRT to assess radiological response to treatment.

MR imaging was performed on 3T MAGNETOM Trio, A Tim System (Siemens Medical Systems ${ }^{\circledR}$, Erlangen, Germany) with a neck array coil. The diagnostic imaging protocol included axial T2weighted sequences with short tau inversion recovery (STIR) from the base of the scull to aortic arch (TR/TE 5010/71 ms, TI $170 \mathrm{~ms}$, flip angle (FA) $70^{\circ}$, receiver bandwidth $287 \mathrm{~Hz} /$ pixel, matrix size $256 \times 256$, slice thickness $3 \mathrm{~mm}$, gap $0.3 \mathrm{~mm}$ and field of view (FOV) $18 \times 18 \mathrm{~cm}$ ). Axial slices that covered the entire primary tumour were selected for DWI and DCE-MR imaging.

DWI images were acquired in axial plane using pulsed spin-echo echo-planar image sequence (TR/ TE 3600/86 ms, receiver bandwidth 1302 Hz/pixel, matrix size 180 x 192, slice thickness 5 mm, gap 1.5 $\mathrm{mm}$, FOV $230 \mathrm{~cm}^{2}$ and total acquisition time 5.04 min). Five different $b$-values $(b=0,100,200,500$ and $1000 \mathrm{~s} / \mathrm{mm}^{2}$ ) were used with all diffusion-sensitizing gradients applied in three orthogonal directions to obtain trace-weighted images.

DCE-MRI was performed using 3D fast low angle shot (FLASH) sequence optimised for spatial and temporal resolution (TR/TE 5/1.16 ms, FA 15', receiver bandwidth $490 \mathrm{~Hz} /$ pixel, matrix size 220 
$\mathrm{cm}^{2}$, slice thickness $4 \mathrm{~mm}$, temporal resolution $4 \mathrm{~s}$, total acquisition time $5 \mathrm{~min}$. T1 mapping was used to convert signal intensities into gadolinium concentration. The T1 map was calculated from precontrast multiple flip angle images $\left(6^{\circ}, 10^{\circ}\right.$ in $\left.15^{\circ}\right)$. A k-space weighted image contrast algorithm was used to generate images with full spatial resolution of $128 \times 128$. Baseline images were acquired for 28 s. While the imaging continued, the gadobutrol (Gadovist $\AA$, Bayer HealthCare Pharmaceuticals) was administered intravenously in a dose of 0.1 $\mathrm{mmol} / \mathrm{kg}$ body weight with a flow-rate of $3.5 \mathrm{ml} / \mathrm{s}$ followed by a $20 \mathrm{ml}$ of saline flush with power injector.

Finally, post-contrast axial T1 weighted volumetric interpolated breath-hold examination (VIBE) sequences were acquired from skull base to aortic arch (TR/TE 3,26/1,26 ms, voxel size 1,1 x $0,9 \times 1,5 \mathrm{~mm}$ with $4 \mathrm{~mm}$ averages, receiver bandwidth $640 \mathrm{~Hz} /$ pixel, matrix size 218 x 288 and FOV $\left.250 \mathrm{~cm}^{2}\right)$.

\section{Imaging parametrs analysis}

Post-processing of all the images was performed at a workstation running commercially available software Olea Sphere ${ }^{\circledR}$ 3.0 MR Head \& Neck expanded applications (Olea Medical ${ }^{\circledR}$, La Ciotat, France). Before data analysis, motion correction algorithm was applied that allowed for pairwise in-plane (acquisition plane) rigid co-registration of all raw perfusion images of a given slice location with wellchosen reference image over time.

Quantitative DCE-MRI parameters were volume transfer constant $\left(\mathrm{K}_{\text {trans }}\right)$, extracellular extravascular volume fraction $\left(\mathrm{v}_{\mathrm{e}}\right)$ and plasma volume fraction $\left(\mathrm{V}_{\mathrm{p}}\right)$. The pharmacokinetic modelling was done on a pixel-by-pixel basis using the extended Tofts model - a two compartment model, which is suitable for any freely diffusible tracer. The equation modelling the contrast agent's concentration was the following ${ }^{17}$ :

$C(t)=v_{p} c_{p}(t)+K_{\text {trans }} c_{p}(t) x \exp \left(-K_{e p} t\right)$

where $C(t)$ is the tissue contrast agent concentration time course, $\mathrm{V}_{\mathrm{p}}$ is plasma volume fraction, $c_{p}$ is plasma contrast agent concentration, $K_{\text {trans }}$ is the volume transfer constant and $\mathrm{K}_{\mathrm{ep}}$ is the transfer function from extracellular extravascular space to the plasma space. The Arterial Input Function (AIF) for the pharmacokinetic analysis was derived from automatic selection of perfusion weighted image pixels selected in suitable arteries using a dedicated algorithm. The following multi-parametric maps with $\mathrm{K}_{\text {trans' }}, \mathrm{V}_{\mathrm{e}}$ and $\mathrm{V}_{\mathrm{p}}$ were then obtained automatically.

Apparent diffusion coefficient (ADC) maps were computed by a single exponential fit using the DW signal intensity-b value curves.

ADC and DCE-MRI derived parameters as well as tumour volumes were measured at baseline and after $10 \mathrm{~Gy}$. A radiologist (three years of experience in head and neck imaging) placed three regions of interest (ROIs) and analysed the multi-parametric ADC and DCE maps independently, with the T1 post-contrast images serving as the reference images. The ROIs were drawn manually on all imaging sections encircling solid-appearing portions of primary tumours and metastatic lymph node or nodal masses. Special attention was paid not to include necrotic and cystic areas (hyper-intense areas on STIR images), large feeding vessels and surrounding normal tissue.

Tumour volumes were calculated by manually encircling tumour borders on each $\mathrm{T} 1$ post-contrast sequence on all axial slices. Soft wear algorithm then calculated the volume by using the equation:

$V=$ encircled area $x$ section thickness

Mean value of the selected ROIs from primary tumour was calculated for each parameter at each time point. Relative changes of the ADC and DCEMRI parametric values as well as tumour volumes were calculated by dividing the mathematical difference of the corresponding parametric and tumour volume values after 10Gy and at pre-treatment by the pre-treatment parameter value for individual patient using the formula:

$\Delta P_{10 G y}=\left[\frac{P_{10 G y}-P_{0}}{P_{0}}\right] * 100$

where $\mathrm{P}$ represents any given parameter (ADC, $\mathrm{K}_{\text {trans }}, \mathrm{V}_{\mathrm{e}^{\prime}} \mathrm{V}_{\mathrm{p}}$ or tumour volume), $\mathrm{P}_{10 \mathrm{~Gy}}$ represents absolute value of the parameter after $10 \mathrm{~Gy}$, and $\mathrm{P}_{0}$ absolute value of the pre-treatment parameter.

\section{Outcome determination and statistical analysis}

All data analysis and graphs were performed using statistical software SPSS 19.0 (IBM SPSS Statistics for Windows, Version 19.0. Armonk, NY: IBM Corp.). Shapiro-Wilk test and Levene test were used for normality testing and homogeneity-ofvariance testing, respectively. The continuous variables are presented by median value and range. The relative changes in parameters between pretreatment values and at $10 \mathrm{~Gy}$ are expressed in percentage (\%). OS (event: death from any cause) 
TABLE 1. The baseline clinical characteristics of all the patients

\begin{tabular}{cccccc}
\hline Patient & Sex & Age (yrs) & Tumour location & TNM & Stage \\
\hline 1 & $M$ & 53 & Oropharynx & T4aN1 & IVA \\
2 & $M$ & 67 & Hypopharynx & T3N1 & III \\
3 & $M$ & 66 & Hypopharynx & T3N2b & IVA \\
4 & $M$ & 56 & Oropharynx & T3N2b & IVA \\
5 & $M$ & 49 & Oropharynx & T2N2b & IVA \\
6 & $M$ & 57 & Oropharynx & T4aN2c & IVA \\
7 & $M$ & 59 & Hypopharynx & T4aN2b & IVA \\
8 & $M$ & 60 & Oropharynx & T3N2b & IVA \\
9 & $M$ & 58 & Oropharynx & T4aN2b & IVA \\
10 & $M$ & 53 & Oropharynx & T4aN2c & IVA \\
11 & $M$ & 64 & Oropharynx & T3N2c & IVA \\
12 & $M$ & 56 & Hypopharynx & T3N2b & IVA \\
13 & $M$ & 65 & Oropharynx & T2N2b & IVA \\
14 & $M$ & 53 & Hypopharynx & T4aN2c & IVA \\
15 & $M$ & 66 & Oropharynx & T3N2b & IVA \\
16 & $M$ & 65 & Oropharynx & T3N2a & IVA \\
17 & $M$ & 46 & Oropharynx & T3N3 & IVB \\
18 & $M$ & 67 & Hypopharynx & T3N1 & III \\
19 & M & 58 & Oropharynx & T3N1 & III \\
20 & $M$ & 48 & Hypopharynx & T3N1 & III \\
\hline
\end{tabular}

Yrs = years parameters as well as tumour volume and clinical parameters for predicting survival rates. Area under curve (AUC) was computed and the optimal cut-off values were calculated by maximizing sensitivity and specificity. Two-tailed $p$ values less than 0.05 were considered statistically significant.

\section{Results}

\section{Patient data}

All of the 20 consecutive patients (19 men, 1 woman; median age 58 years, range $46-67$ years) with loco-regionally advanced HNSCC (oropharynx 13, hypopharynx 7) were free of systemic disease at presentation. The baseline clinical characteristics of the patients are listed in Table 1.

The median follow-up times of the entire study cohort and of the surviving patients were 27.2 months (range, 2.6-51.4 months) and 30.2 months (range, 18.2-51.4 months), respectively. In all patients, radiological complete tumour response to cCRT was determined 2.5-3 months post-therapy. Disease reappearance was diagnosed in 5 patients: $3(15 \%)$ patients had recurrence at primary site, and 2 patients $(10 \%)$ developed distant metastases (lungs). At the time of analysis, five patients (25\%) were dead. Locoregional disease was the cause of death in three patients (with survival rates of 5.2, 10.6, and 22.9 months post-therapy), distant metastasis in one patient (14.1 months) and acute lower respiratory tract infection in the remaining patient (2.6 months, considered as treatment failure). A mean DFS was 38.9 months (2.6-51.4 months, 95\% Confidence Interval [CI] 30.7-47.1). A mean OS time was 40.6 months (2.6-51.4 months, 95\% CI 32.4-48.8). The 1- and 2- year DFS survival rates were $88.8 \%$ and $88.2 \%$ and for OS they were $84.4 \%$ and $73.8 \%$.

\section{Descriptive analysis of ADC and DCE-MRI derived parameters before and during treatment}

For all the included patients, the pre-treatment values of ADC and DCE-MRI derived parameters $\mathrm{K}_{\text {trans' }}, \mathrm{V}_{\mathrm{e}}$ and $\mathrm{V}_{\mathrm{p}}$ were $0.81(0.62-1.07) \times 10^{-3} \mathrm{~mm}^{2} / \mathrm{s}$, $0.48(0.13-0.79) \mathrm{min}^{-1}, 0.32(0.15-0.64)$ and 0.17 (0.06-0.43), respectively. A statistically significant change from pre-treatment to $10 \mathrm{~Gy}$ was seen in all parameters: an increase of $22.5 \%$ (1.7 to $52.4 \%$ ) in ADC ( $p<0.001)$ and of $70 \%(73.2$ to $357.14 \%)$ in $\mathrm{V}_{\mathrm{p}}(\mathrm{p}=0.015)$ and a reduction of $50.3 \%(-93.4$ to $179.2 \%)$ in $\mathrm{K}_{\text {trans }}(\mathrm{p}=0.003)$ and of $35.2 \%(-88.9$ 
TABLE 2. Absolute values of DWI and DCE-MRI derived parameters and tumour volumes before treatment and their relative changes after 10 Gy (expressed as median and range) for the entire cohort of the patients and separately for alive and deceased patients at the end of follow-up

\begin{tabular}{|c|c|c|c|}
\hline Parameters & $\begin{array}{l}\text { The entire cohort of patients } \\
\qquad(n=20,100 \%)\end{array}$ & $\begin{array}{l}\text { Patients still alive at the end } \\
\text { of follow-up } \\
(n=15 ; 75 \%)\end{array}$ & $\begin{array}{l}\text { Deceased patients at the end } \\
\text { of follow-up } \\
(n=5 ; 25 \%)\end{array}$ \\
\hline \multicolumn{4}{|c|}{ Pre-treatment values } \\
\hline $\mathbf{K}_{\text {trans }}\left(\min ^{-1}\right)$ & $0.48(0.13-0.79)$ & $0.57(0.23-0.79)$ & $0.22(0.13-0.35)$ \\
\hline $\mathbf{v}_{\mathrm{e}}$ & $0.32(0.15-0.64)$ & $0.32(0.17-0.64)$ & $0.22(0.15-0.37)$ \\
\hline Tumour volume (ml) & $10.5(2.6-54.9)$ & $8.8(2.6-44.55)$ & $18.21(6.6-54.9)$ \\
\hline \multicolumn{4}{|c|}{ Relative changes after $10 \mathrm{~Gy}$} \\
\hline$\triangle \mathrm{ADC}$ & $22.5 \%$ (1.7 to $52.4 \%)$ & $25.0(4.7$ to 51.4$)$ & 9.2 (1.7 to 52.4$)$ \\
\hline$\Delta \mathrm{K}_{\text {trans }}$ & $-50.3 \%$ (-93.4 to $179.2 \%)$ & $-60.0(-93.0$ to -43.2$)$ & $4.2(-37.1$ to 179.2$)$ \\
\hline$\Delta \mathrm{V}_{\mathrm{e}}$ & $-35.2 \%(-88.9$ to $126.8 \%)$ & $-40.0(-88.9$ to 65.4$)$ & $-14.7(-40$. to 126.8$)$ \\
\hline
\end{tabular}

$\mathrm{ADC}=$ apparent diffusion coefficient; $\mathrm{DCE}-\mathrm{MRI}=$ dynamic contrast-enhanced $\mathrm{MRI}$; DWI = diffusion weighted imaging; $\mathrm{Gy}=$ gray; $\mathrm{K}_{\text {trans }}=\mathrm{volume}$ transfer constant; $\mathrm{V}_{\mathrm{e}}=$ extracellular extravascular volume fraction; $V_{p}=$ plasma volume fraction; $\Delta=$ relative change in the parameter

to $126.8 \%)$ in $\mathrm{V}_{\mathrm{e}}(\mathrm{p}=0.024)$. Pre-treatment tumour volume was $10.5 \mathrm{ml}(2.6-54.9 \mathrm{ml})$. However, the change after $10 \mathrm{~Gy}$ from pre-treatment was not statistically different $(-15.0 \% ;-76.3$ to $254.6 \%$; $\mathrm{p}=$ 0.823) (Table 2).

The values of ADC and DCE-MRI derived parameters before treatment and the relative change of parameters after $10 \mathrm{~Gy}$ from baseline were com- pared between patients alive at the end of followup and those who died (Table 2). Pre-treatment $\operatorname{ADC}\left(0.76 \times 10^{-3} \mathrm{~mm}^{2} / \mathrm{s} ; 0.62-0.91 \times 10^{-3} \mathrm{~mm}^{2} / \mathrm{s}\right)$ was significantly lower $(p=0.042)$ in patients which were still alive at the end of follow-up versus the patients who died $\left(0.96 \times 10^{-3} \mathrm{~mm}^{2} / \mathrm{s} ; 0.76-1.07 \times\right.$ $10^{-3} \mathrm{~mm}^{2} / \mathrm{s}$ ). Alive patients also showed a significantly higher $(p=0.002)$ pre-treatment DCE-MRI

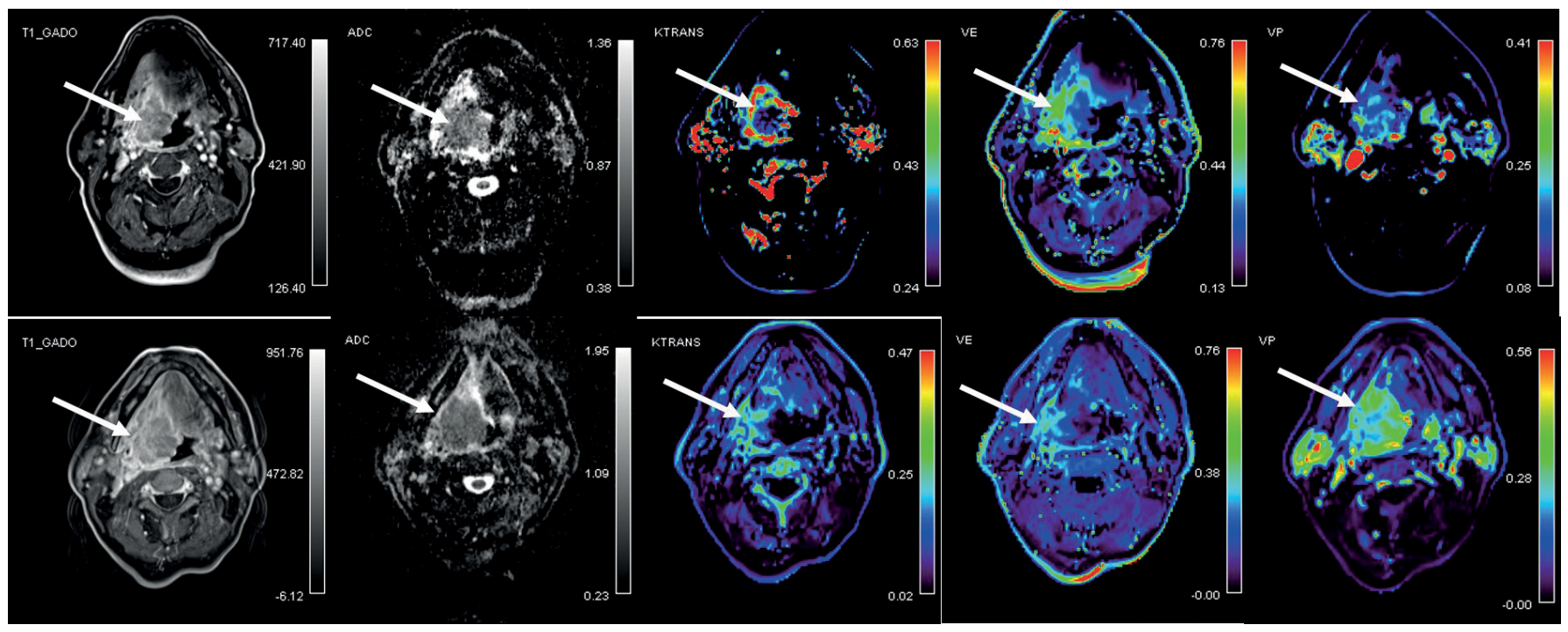

FIGURE 1. 58 year old patient with oropharyngeal squamous cell carcinoma (T4a N2b) who was alive at the end of follow up with overall survival of 41.1 months. Axial post-contrast Tl images, co-registred and corresponding apparent diffusion coefficient (ADC) maps and color-coded dynamic contrastenhanced MRI derived volume transfer constant (Ktrans), extracellular extravascular fraction (Ve) and plasma volume fraction (Vp) maps in the area of the tumour (arrows) are shown. Top: before treatment; bottom: after 10 Gray of chemo-radiotherapy. 


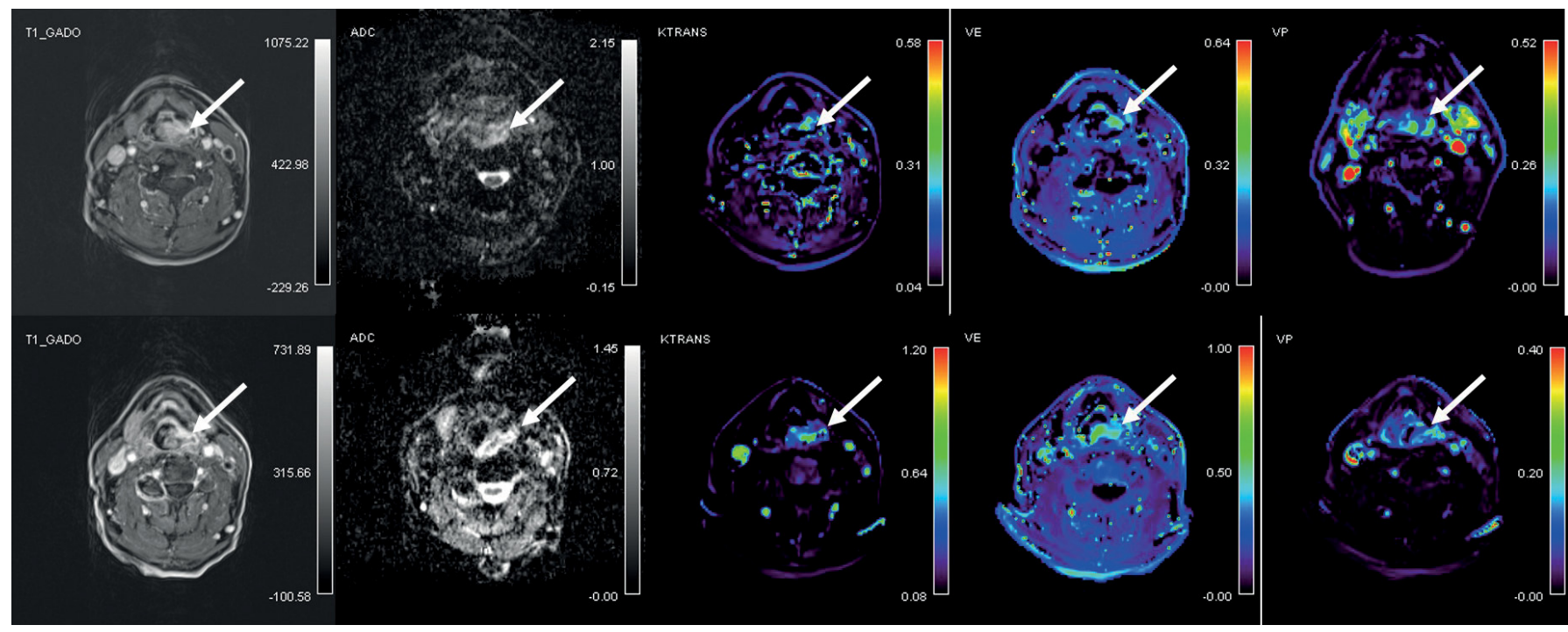

FIGURE 2. 65 year old patient with hypopharyngeal squamous cell carcinoma (T2 N2) who died 14 months of starting of chemo-radiotherapy due to disease relaps into the lungs. Axial post-contrast Tl images, co-registred and corresponding apparent diffusion coefficient (ADC) maps and colorcoded dynamic contrast-enhanced MRI derived volume transfer constant (Ktrans), extracellular extravascular fraction (Ve) and plasma volume fraction (Vp) maps in the area of the tumour (arrows) are shown. Top: before treatment; bottom: after 10 Gray of chemo-radiotherapy.

derived parameter $\mathrm{K}_{\text {trans }}\left(0.57 \mathrm{~min}^{-1} ; 0.23-0.79 \mathrm{~min}^{-}\right.$ $\left.{ }^{1}\right)$ compared with patients that died during followup $\left(0.22 \mathrm{~min}^{-1} ; 0.13-0.35 \mathrm{~min}^{-1}\right)$. In addition, alive patients demonstrated a reduction in $\mathrm{K}_{\text {rans }}$ after 10

TABLE 3. Univariate analysis of risk factors associated with disease-free survival and overall survival rates $(n=20)$

\begin{tabular}{|c|c|c|}
\hline Parameters & $\begin{array}{c}\text { Disease-free survival } \\
\text { ( } p \text { value })\end{array}$ & $\begin{array}{l}\text { Overall survival } \\
\text { ( } p \text { value) }\end{array}$ \\
\hline \multicolumn{3}{|c|}{ Clinical parameters } \\
\hline Age & 0.338 & 0.556 \\
\hline Tumour location & 0.396 & 0.752 \\
\hline Stage & 1.000 & 0.198 \\
\hline \multicolumn{3}{|c|}{ Pre-treatment values of functional parameters and tumour volume } \\
\hline $\operatorname{ADC}\left(\times 10^{-3} \mathrm{~mm}^{2} / \mathrm{s}\right)$ & 0.856 & $0.012^{*}$ \\
\hline$K_{\text {trans }}\left(\mathrm{min}^{-1}\right)$ & 0.116 & $0.026^{*}$ \\
\hline$\vee_{e}$ & 0.754 & 0.293 \\
\hline$V_{p}$ & 0.165 & 0.342 \\
\hline Tumour volume & 0.959 & $0.048^{*}$ \\
\hline \multicolumn{3}{|c|}{ Relative changes after $10 \mathrm{~Gy}$ of functional parameters and tumour volume } \\
\hline$\triangle \mathrm{ADC}(\%)$ & 0.740 & 0.061 \\
\hline$\Delta \mathrm{K}_{\text {trans }}$ & 0.688 & $0.014^{*}$ \\
\hline$\Delta \mathrm{V}_{\mathrm{e}}$ & 0.957 & 0.405 \\
\hline$\Delta \mathrm{V}_{\mathrm{p}}$ & 0.672 & 0.077 \\
\hline$\Delta$ Tumour volume $(\mathrm{ml})$ & 0.495 & 0.486 \\
\hline
\end{tabular}

* = significant predictor at univariate analysis; $A D C=$ Apparent diffusion coefficient; $\mathrm{K}_{\text {trans }}=$ volume transfer constant; $\vee_{e}=$ extracellular extravascular volume fraction; $V_{p}=$ plasma volume fraction; $\triangle$

$=$ relative change in the parameter
Gy from baseline (-60.0\%; -93.0 to $-43.2 \%)$ whereas the patients who died showed no change or an increase in $\mathrm{K}_{\text {rans }}(4.2 \% ;-37.1$ to $179.2 \%)(\mathrm{p}<0.001)$. There was no difference in the pre-treatment values of other perfusion parameters and tumour volumes $(p>0.05)$ nor in the relative changes from 10 Gy to baseline of ADC, $V_{e^{\prime}} V_{p}$ and tumour volume ( $p>0.05)$. Illustrative examples of DW and DCE MRI images of alive and deceased patient at the end of follow up are shown in Figure 1 and 2, respectively.

On contrary, no significant difference was observed when values of all the functional parameters and tumour volumes where compared between patients that showed disease progression during follow-up and patients without disease progression $(p>0.05)$. However, in patients with noted disease progression there was a trend of larger pretreatment tumour volume $(14.0 \mathrm{ml} ; 8.9-55.0 \mathrm{ml})$ compared to the group with disease controlled (6.6 $\mathrm{ml} ; 2.6-50.8 \mathrm{ml})(\mathrm{p}=0.054)$.

\section{Univariate and multivariate Cox regression analysis}

When assessing potential prognostic factors for DFS, none of them reached the level of statistical significance on univariate analysis (Table 3). Pretreatment parameters that significantly influenced OS on univariate analysis were ADC ( $\mathrm{p}=0.012)$, $\mathrm{K}_{\text {trans }}(\mathrm{p}=0.026)$, and tumour volume $(\mathrm{p}=0.048)$ 


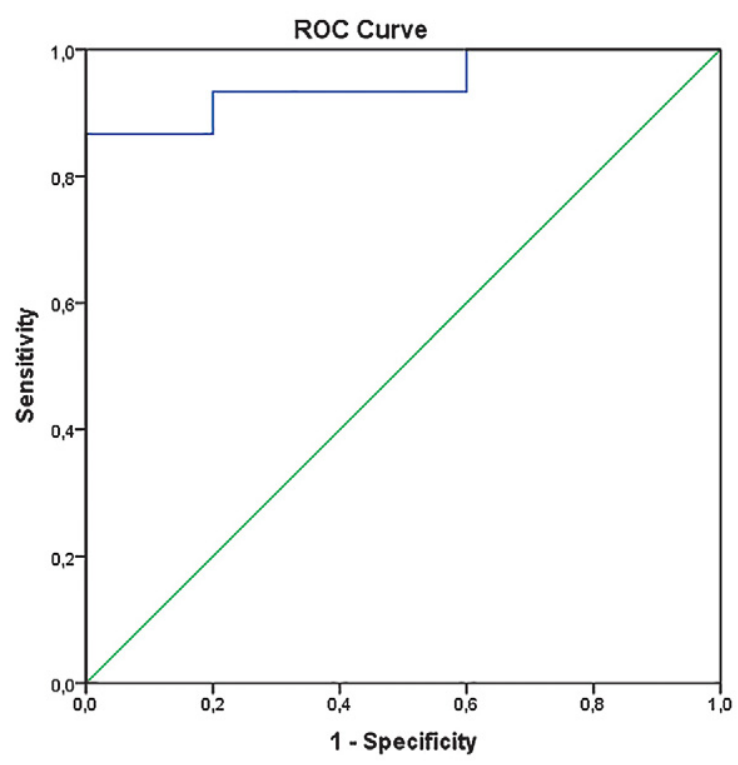

FIGURE 3. Receiver operating characteristic (ROC) curves for dynamic contrast-enhanced MRI (DCE-MRI) derived parameter $\mathrm{K}_{\text {trans }}$ (volume transfer constant) exhibiting area under ROC curve (AUC) of 0.95 with sensitivity $93.3 \%$ and specificity $80 \%$. $(p=0.003)$.

(higher $\mathrm{K}_{\text {trans }}$ and lower ADC and tumour volume were associated with longer survival). In addition, higher reduction in $\mathrm{K}_{\text {trans }}(\mathrm{p}=0.014)$ from baseline to $10 \mathrm{~Gy}$ was also identified as a significant prognosticator for OS but not also any of the clinical parameters (age, tumour location, TNM stage). Multivariate Cox regression analysis identified only higher pre-treatment $\mathrm{K}_{\text {trans }}(\mathrm{p}=0.026$; $95 \% \mathrm{CI}$ : $0.000-0.132$ ) as an independent predictor of OS.

\section{ROC curve analysis}

When ROC curve analysis was performed, pretreatment $\mathrm{K}_{\text {trans. }}$ provided an excellent diagnostic accuracy with an area under curve (AUC) of 0.95 $(\mathrm{p}=0.003 ; 95 \% \mathrm{CI}: 0.85-1.00)$ and a sensitivity and specificity of $93.3 \%$ and $80.0 \%$, respectively (Figure 1). The cut-off value for longer survival was set at $\geq 0.29 \mathrm{~min}^{-1}$. In addition, a reduction in $\mathrm{v}_{\mathrm{e}}$ after 10 Gy (survivors: median $-40.0 \%$, range $-88.9 \%$ to $65.4 \%$; deceased patients: median $-14.7 \%$, range -40.0 to $126.8 \%$ ) yielded a good diagnostic accuracy with AUC 0.80 ( $p=0.055$; 95\% CI: 0.569-1.000), sensitivity $73.3 \%$ and specificity $80.0 \%$. Pre-treatment $\mathrm{v}_{\mathrm{e}}$ showed only poor diagnostic usefulness accuracy (AUC 0.63), whereas other parameters failed to show any diagnostic accuracy $(A U C<0.6)$. When patients were grouped according to the $\mathrm{K}_{\text {trans }}$ cutoff value of $0.29 \mathrm{~min}^{-1}$, the log rank test confirmed

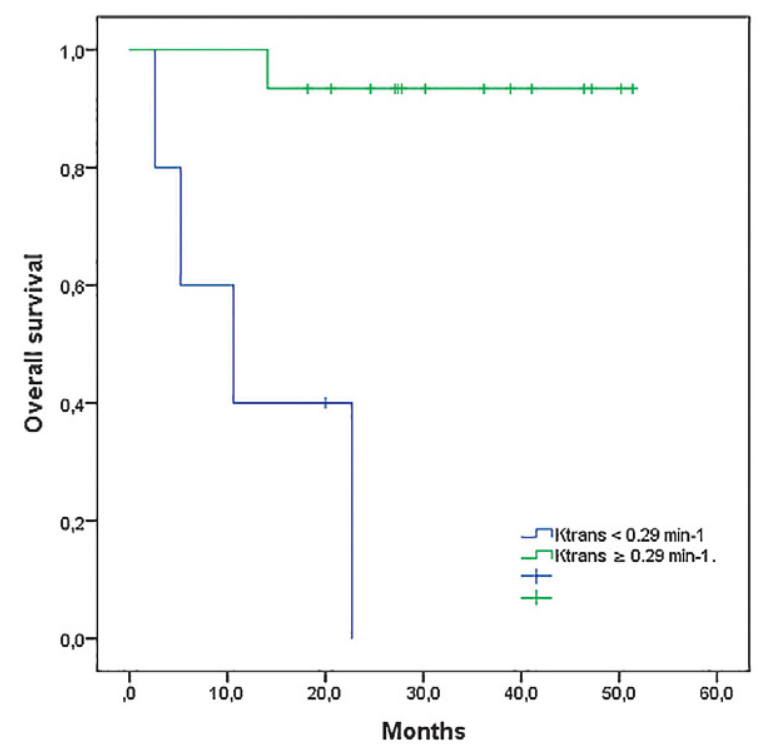

FIGURE 4. Kaplan-Meier estimates of overall survival in HNSCC patients (20) stratified according to pre-treatement DCEMRI derived parameter $K_{\text {trans }}$ (cutt-off value of $\geq 0.29 \mathrm{~min}^{-1}$, determined at ROC curve analysis).

DCE-MRI = dynamic contrast-enhanced MRI; $K_{\text {trans }}=$ volume transfer constant; $R O C=$ receiver operating curve

a statistically significant difference in the length of OS between the two groups with mean survival of 48.9 months (95\% CI: 44.2-53.6) in patients with pre-treatment $\mathrm{K}_{\text {trans }} \geq 0.29 \mathrm{~min}^{-1}$ and 12.8 months (95\% CI: 4.1-21.4) in patients with pre-treatment $\mathrm{K}_{\text {trans }}<0.29 \mathrm{~min}^{-1}(\mathrm{p}<0.001)$ (Figure 3$)$.

\section{Discussion}

Our study showed that pre-treatment DCE-MRI derived parameter $\mathrm{K}_{\text {trans }}$ could serve as an independent prognosticator of OS. Thus, DCE-MRI may be used before and early during treatment to get insight into tumour vascularity and perfusion, which conditioned, among other biological factors, the response of the HNSCC to platin-based cCRT. The ability to identify specific pre-treatment parameters as well as their changes early during treatment could assist in identification of patients at higher recurrence and mortality risk. Therefore, re-consideration of existing treatment scenario might be indicated in such patients in order to maximize the chance of favourable outcome and to avoid unnecessary toxicity. ${ }^{18,19}$ In addition, more intensive surveillance could be performed in those individuals to detect eventual treatment failure in a timely manner. ${ }^{19}$ 
In present study, pre-treatment $\mathrm{K}_{\text {trans }}$ was found to be an independent prognostic factor for OS in both univariate and multivariate analysis with an excellent diagnostic accuracy in ROC analysis. DCE-MRI allows the analysis of vascular-related parameters in tumours and has primarily been used to predict treatment response and loco-regional control in HNSCC patients. ${ }^{20-25}$ Very few studies evaluated the role of pre-treatment DCE-MRI on the survival of HNSCC patients treated with cCRT. Chan and Chawla with co-workers found that lower pre-treatment $\mathrm{K}_{\text {trans }}$ is associated with poorer DFS and OS. ${ }^{21,26}$ On the contrary, two studies by $\mathrm{Ng}$ et al. failed to show the prognostic role of $\mathrm{K}_{\text {trans }}$ for survival in oropharyngeal HNC treated with CRT. Previous studies have already shown that higher pre-treatment $K_{\text {trans }}$ values indicate better tumour response $\mathrm{e}^{20,22}$ and loco-regional control to CRT. ${ }^{24} \mathrm{~K}_{\text {trans }}$ is the volume transfer constant between the blood plasma and extracellular extravascular space and is a measure of both tumour blood flow and vascular permeability. Studies on different tumours found that $\mathrm{K}_{\text {trans }}$ correlates with the proliferating cell density and micro-vessel density (MVD). ${ }^{13,27,28}$ Both are used as markers of angiogenesis which is supposed to be the leading process for tumour growth, metastasis and radiosensitivity. ${ }^{29}$ The leading factor for stimulating angiogenesis is hypoxia, caused by structurally and functionally abnormal vessels and higher oxygen consumption by fast proliferating cells within the tumour micro-environment. ${ }^{30}$ It is well-known that hypoxia enhances radio-chemoresistance through several mechanisms. ${ }^{31}$ Higher pre-treatment $\mathrm{K}_{\text {trans }}$ may therefore reflect greater tumour blood flow and vessels permeability, indicating enriched oxygenation that resulted in favourable radio-chemosensitivity status of the tumour.

Although the multivariate analysis failed to confirm an independent prognostic value of relative changes of $\mathrm{K}_{\text {trans' }}$ a higher reduction of $\mathrm{K}_{\text {trans }}$ after 10 Gy from baseline was found to be important for better OS in univariate analysis. Studies addressing the prognostic values on survival rates of DCEMRI early during treatment are lacking. Kim et al. reported a reduction of $\mathrm{K}_{\text {trans }}$ in human head and neck tumour xenografts after only three days of RT or chemotherapy. ${ }^{13}$ Conversely, Baer et al. reported that patients with large-volume HNSCC with decreased $\mathrm{K}_{\text {trans }}$ after two weeks of therapy may have a shorter survival period. ${ }^{15}$ Aforementioned study by Kim et al. showed that reduction of $\mathrm{K}_{\text {trans }}$ correlates with the changes of both proliferating cell density and MVD and the authors postulated that repopulation of RT-resistant endothelial cells after first days of CRT may be faster in more radiosensitive tumours leading to lower permeability. ${ }^{13}$ Therefore, successful treatment leads to lower tumour perfusion and permeability, thus reflecting as a reduction of $\mathrm{K}_{\text {trans }}$.

In addition, lower pre-treatment ADC was found to be a significant factor for longer survival at univariate analysis, but was not significant when multivariate analysis was applied. This is in agreement with Chan et al..$^{26}$ and $\mathrm{Ng}$ et al. ${ }^{32}$ who also found no significant prognostic value of pre-treatment ADC for 3-year OS in HNSCC treated with CRT. On the contrary, lower pre-treatment ADC was shown to be a consistent predictor of higher DFS of head and neck cancer to CRT. ${ }^{33,34}$ DW MRI is a quantitative imaging technique that measures diffusion of water molecules. Thus, in tissues with high cellularity (e.g. tumours), diffusion of water molecules is limited, showing as low ADC. Driessen et al. demonstrated that tumours with higher pre-treatment ADC had poorer prognosis due to lower cellularity and higher proportion of stroma that is a known independent poor prognostic factor. ${ }^{35,36}$ However, ADC value is probably affected by various physiologic parameters other than tumour cellularity and that needs to be explored further. ${ }^{37}$

Our study has several limitations which have to be addressed. The main one is the small sample size and high number of parameters to analyse. However, our series is relatively homogeneous concerning primary tumour site and histological characteristics when compared to other studies conducted in HNSCC. This could explain why ADC or majority of DCE-MRI parameters failed to show any prognostic role when assessing $O S$ and why none of the parameters was predictive for DFS. Larger prospective studies with higher number of patients and longer follow-up are needed in order to address the true prognostic role of DW and DCE-MRI derived parameters. Second, the DWI- and DCE-MRI-derived parameters were calculated as a mean value from the three most representative ROIs, delineated by only one radiologist. Therefore, no interobserver variability could be analysed, and appropriateness of ROIs marking procedure can also be questioned. Another limitation was that the DCE-MRI derived parameters and ADC were not compared to well-established histological biomarkers of angiogenesis (e.g. CD31 and Ki67 expressing cells). The last drawback can be attributed to the patient-specific AIF measurement as was used in our study: according to relevant studies, the use of a population-based AIF is 
recommended due to most favourable repeatability. ${ }^{38}$

In conclusion, the results of our study show that pre-treatment $\mathrm{K}_{\text {trans }}$ may serve as a biomarker of tumour angiogenesis. Therefore, DCE-MRI could play a role in determining prognosis of HNSCC patient treated with platin-based cCRT.

\section{Acknowledgments}

The authors thank Ass. Prof. Sotirios Bisdas, M.D., Ph.D., of University College London Hospitals NHS for help with establishing the DCE- and DWIMR protocols.

\section{References}

1. Ferlay J, Soerjomataram I, Dikshit R, Parkin DM, Forman D, Bray F. Cancer incidence and mortality worldwide: sources, methods and major patterns in GLOBOCAN 2012. Int J Cancer 2015; 136: E359-86. doi: 10.1002/ijc.29210

2. Strojan P. [New approaches to radiotherapy of head and neck tumors]. [Slovenian]. Zdr Vestn 2010; 79: 339-53.

3. Winquist E, Agbassi C, Meyers BM, Yoo J, Chan KKW. Systemic therapy in the curative treatment of head and neck squamous cell cancer: a systematic review. J Otolaryngol Head Neck Surg 2017; 46: 29. doi: 10.1186/s40463017-0199-x

4. Leeman JE, Li J, Pei X, Venigalla P, Zumsteg ZS, Katsoulakis E, et al. Patterns of treatment failure and postrecurrence outcomes among patients with locally advanced head and neck squamous cell carcinoma after chemoradiotherapy using modern radiation techniques. JAMA Oncol 2017; 3: 1487-94. doi: 10.1001/jamaoncol.2017.0973

5. Pignon J-P, Maître A le, Maillard E, Bourhis J. Meta-analysis of chemotherapy in head and neck cancer (MACH-NC): an update on 93 randomised trials and 17,346 patients. Radiother Oncol 2009; 92: 4-14. doi: 10.1016/j. radonc 2009.04 .014

6. Brizel DM, Prosnitz RG, Hunter S, Fisher SR, Clough RL, Downey MA, et al. Necessity for adjuvant neck dissection in setting of concurrent chemoradiation for advanced head-and-neck cancer. Int J Radiat Oncol 2004; 58: 141823. doi: 10.1016/j.ijrobp.2003.09.004

7. Blanchard P, Baujat B, Holostenco V, Bourredjem A, Baey C, Bourhis J, et al; $\mathrm{MACH}-\mathrm{CH}$ Collaborative group. Meta-analysis of chemotherapy in head and neck cancer (MACH-NC): a comprehensive analysis by tumour site. Radiother Oncol 2011; 100: 33-40. doi: 10.1016/j.radonc.2011.05.036

8. Bisdas S, Rumboldt Z, Surlan-Popovic K, Baghi M, Koh TS, Vogl TJ, et al. Perfusion CT in squamous cell carcinoma of the upper aerodigestive tract: long-term predictive value of baseline perfusion CT measurements. AJNR Am J Neuroradiol 2010; 31: 576-81. doi: 10.3174/ajnr.A1852

9. Surlan-Popovic, K. Bisdas S, Rumboldt Z, Koh TS, Strojan P. Changes in perfusion $C T$ of advanced squamous cell carcinoma of the head and neck treated during the course of concomitant chemoradiotherapy. Am J Neuroradiol 2010; 31: 570-5. doi: 10.3174/ajnr.A1859

10. Pietsch C, de Galiza Barbosa F, Hullner MW, Schmid DT, Haerle SK, Huber GF, et al. Combined PET/CT-perfusion in patients with head and neck cancers might predict failure after radio-chemotherapy: a proof of concept study. BMC Med Imaging 2015; 15: 60. doi: 10.1186/s12880-015-0102-z

11. Koh D-M, Lee J-M, Bittencourt LK, Blackledge M, Collins DJ. Body diffusionweighted MR imaging in oncology. Magn Reson Imaging Clin N Am 2016 24: 31-44. doi: 10.1016/j.mric.2015.08.007

12. Gaddikeri S, Gaddikeri RS, Tailor T, Anzai Y. Dynamic contrast-enhanced MR imaging in head and neck cancer: techniques and clinical applications. AJNR Am J Neuroradiol 2016; 37: 588-95. doi: 10.3174/ajnr.A4458
13. Kim H, Hartman YE, Zhai G, Chung TK, Korb ML, Beasley TM, et al. Dynamic contrast-enhanced MRI evaluates the early response of human head and neck tumor xenografts following anti-EMMPRIN therapy with cisplatin or irradiation. J Magn Reson Imaging 2015; 42: 936-45. doi: 10.1002/jmri.24871

14. Cao Y, Popovtzer A, Li D, Chepeha DB, Moyer JS, Prince ME, et al. Early prediction of outcome in advanced head-and-neck cancer based on tumor blood volume alterations during therapy: a prospective study. Int J Radiat Oncol Biol Phys 2008; 72: 1287-90. doi: 10.1016/j.jijrobp.2008.08.024.EARLY

15. Baer AH, Hoff BA, Srinivasan A, Galbán CJ, and Mukherji SK. Feasibility analysis of the parametric response map as an early predictor of treatment efficacy in head and neck cancer. AJNR Am J Neuroradiol 2015; 36: 757-62. doi: 10.3174/ajnr.A4296

16. Strojan $P$, Zadnik V, Šifrer R, Lanišnik B, Didanović V, Jereb $S$, et al. Incidence trends in head and neck squamous cell carcinoma in Slovenia, 1983-2009: role of human papillomavirus infection. Eur Arch Otorhinolaryngol 2015; 272: 3805-14. doi: 10.1007/s00405-014-3459-7

17. Tofts PS, Brix G, Buckley DL, Evelhoch JL, Henderson E, Knopp MV et al. Estimating kinetic parameters from dynamic contrast-enhanced $\mathrm{T}(1)$-weighted MRI of a diffusable tracer: standardized quantities and symbols. J Magn Reson Imaging 1999; 10: 223-32. doi: 10.1002/(SICI)15222586(199909)10:3<223::AID-JMRI2>3.0.CO;2-S

18. Schwartz DL, Garden AS, Thomas J, Chen Y, Zhang Y, Lewin J, et al. Adaptive radiotherapy for head-and-neck cancer: initial clinical outcomes from a prospective trial. Int J Radiat Oncol Biol Phys 2012; 83: 986-93. doi: 10.1016/j. ijrobp.2011.08.017

19. Yuan J, Lo G, King AD. Functional magnetic resonance imaging techniques and their development for radiation therapy planning and monitoring in the head and neck cancers. Quant Imaging Med Surg 2016; 6: 430-48. doi: 10.21037/qims.2016.06.11

20. Kim S, Loevner LA, Quon H, Kilger A, Sherman E, Weinstein G, et al Prediction of response to chemoradiation therapy in squamous cell carcinomas of the head and neck using dynamic contrast-enhanced MR imaging. AJNR Am J Neuroradiol 2010; 31: 262-8. doi: 10.3174/ajnr.A1817

21. Chawla S, Kim S, Loevner LA, Hwang WT, Weinstein G, Chalian A, et al. Prediction of disease-free survival in patients with squamous cell carcinomas of the head and neck using dynamic contrast-enhanced MR imaging. AJNR Am J Neuroradiol 2011; 32: 778-84. doi: 10.3174/ajnr.A2376

22. Chawla S, Kim S, Dougherty L, Wang S, Loevner LA, Quon H, et al. Pretreatment diffusion-weighted and dynamic contrast-enhanced MR for prediction of local treatment response in squamous cell carcinomas of the head and neck. Am J Roentgenol 2013; 200: 35-43. doi: 10.2214/ AJR.12.9432

23. Chikui T, Kitamoto E, Kawano S, Sugiura T, Obara M, Simonetti AW, et al. Pharmacokinetic analysis based on dynamic contrast-enhanced MRI for evaluating tumor response to preoperative therapy for oral cancer. J Magn Reson Imaging 2012; 36: 589-97. doi: 10.1002/jmri.23704

24. Ng SH, Lin CY, Chan SC, Yen TC, Liao CT, Chang JT, et al. Dynamic contrast-enhanced MR imaging predicts local control in oropharyngeal or hypopharyngeal squamous cell carcinoma treated with chemoradiotherapy. PLoS One 2013; 8: 1-11. doi: 10.1371/journal.pone.0072230

25. Ng SH, Lin CY, Chan SC, Lin YC, Yen TC, Liao CT, et al. Clinical utility of multimodality imaging with dynamic contrast-enhanced MRI, diffusion-weighted MRI, and18F-FDG PET/ CT for the prediction of neck control in oropharyngeal or hypopharyngeal squamous cell carcinoma treated with chemoradiation. PLoS One 2014; 9: 1-19. doi: 10.1371/journal.pone.0115933

26. Chan SC, Cheng NM, Hsieh $\mathrm{CH}$, et al. Multiparametric imaging using ${ }^{18} \mathrm{~F}-\mathrm{FDG}$ $\mathrm{PET} / \mathrm{CT}$ heterogeneity parameters and functional MRI techniques: prognostic significance in patients with primary advanced oropharyngeal or hypopharyngeal squamous cell carcinoma treated with chemoradiotherapy. Oncotarget 2017; 8: 62606-21. doi: 10.18632/oncotarget.15904

27. Surov A, Meyer HJ, Leifels L, Höhn A-K, Richter C, Winter K. Histogram analysis parameters of dynamic contrast-enhanced magnetic resonance imaging can predict histopathological findings including proliferation potential, cellularity, and nucleic areas in head and neck squamous cell carcinoma. Oncotarget 2018; 9: 21070-7. doi: 10.18632/oncotarget.24920

28. Kim SH, Lee HS, Kang BJ, Song BJ, Kim HB, Lee H, et al. Dynamic contrast-enhanced MRI perfusion parameters as imaging biomarkers of angiogenesis. PLOS One 2016; 11: 1-12. doi: 10.1371/journal.pone.0168632 
29. Choi YJ, Lee JH, Kim HO, Kim DY, Yoon RG, Cho SH, et al. Histogram analysis of apparent diffusion coefficients for occult tonsil cancer in patients with cervical nodal metastasis from an unknown primary site at presentation. Radiology 2016; 278: 146-55. doi: 10.1148/radiol.2015141727

30. García-Figueiras R, Padhani AR, Beer AJ, Baleato-González S, Vilanova JC Luna $A$, et al. Imaging of tumor angiogenesis for radiologists - part 1: biological and technical basis. Curr Probl Diagn Radiol 2015; 44: 407-24. doi: 10.1067/j.cpradiol.2015.02.010

31. Manoochehri Khoshinani H, Afshar S, Najafi R. Hypoxia: a doubleedged sword in cancer therapy. Cancer Invest 2016; 34: 536-45. doi: 10.1080/07357907.2016.1245317

32. Ng SH, Liao CT, Lin CY, Chan SC, Lin YC, Yen TC, et al. Dynamic contrast-enhanced MRI, diffusion-weighted MRI and ${ }^{18} \mathrm{~F}$-FDG PET/CT for the prediction of survival in oropharyngeal or hypopharyngeal squamous cell carcinoma treatedwithchemoradiation. Eur Radiol 2016; 26: 4162-72. doi: 10.1007/ s00330-016-4276-8

33. Preda L, Conte G, Bonello L, Giannitto C, Travaini LL, Raimondi S, et al. Combining standardized uptake value of FDG-PET and apparent diffusion coefficient of DW-MRI improves risk stratification in head and neck squamous cell carcinoma. Eur Radiol 2016; 26: 4432-41. doi: 10.1007/ s00330-016-4284-8

34. Noij DP, de Jong MC, Mulders LG, Marcus JT, de Bree R, Lavini C, et al Contrast-enhanced perfusion magnetic resonance imaging for head and neck squamous cell carcinoma: a systematic review. Oral Oncol 2015; 51: 124-38. doi: 10.1016/j.oraloncology.2014.10.016

35. Driessen JP, Caldas-Magalhaes J, Janssen LM, et al. Diffusion-weighted MR imaging in laryngeal and hypopharyngeal carcinoma: association between apparent diffusion coefficient and histologic findings. Radiology 2014; 272 456-63. doi: 10.1148/radiol.14131173

36. Kolenda T, Przybyła W, Kapałczyńska M, Teresiak A, Zajączkowska M, Bliźniak $\mathrm{R}$, et al. Tumor microenvironment - unknown niche with powerful therapeutic potential. Rep Pract Oncol Radiother 2018; 23: 143-53. doi: 10.1016/j. rpor.2018.01.004

37. Choi HS, Kim AH, Ahn SS, Shin NY, Kim J, Lee SK. Glioma grading capability: comparisons among parameters from dynamic contrast-enhanced MR and ADC value on DWI. Korean J Radiol 2013; 14: 487-92. doi: 10.3348/ kjr.2013.14.3.487

38. Rata M, Collins DJ, Darcy J, Messiou C, Tunariu N, Desouza N, et al. Assessment of repeatability and treatment response in early phase clinical trials using DCE-MRI: comparison of parametric analysis using MR- and CT-derived arterial input functions. Eur Radiol 2016; 26: 1991-1998. doi: $10.1007 / \mathrm{s} 00330-015-4012-9$ 